\title{
A systematic review of the cost-effectiveness of uterotonic agents for the prevention of postpartum hemorrhage
}

\author{
Theresa A. Lawrie ${ }^{1,2, *}$ | Ewelina Rogozińska ${ }^{2}$ | Pauline Sobiesuo ${ }^{3}$ | Joshua P. Vogel ${ }^{1,4}$ | \\ Laura Ternent $^{3}$ | Olufemi T. Oladapo ${ }^{1}$
}

\author{
${ }^{1}$ Department of Reproductive Health and \\ Research, UNDP/UNFPA/UNICEF/WHO/ \\ World Bank Special Programme of Research, \\ Development and Research Training in \\ Human Reproduction (HRP), WHO, \\ Geneva, Switzerland \\ ${ }^{2}$ Evidence-Based Medicine Consultancy Ltd, \\ Bath, UK \\ ${ }^{3}$ Health Economics Group, Institute of \\ Health and Society, Newcastle University, \\ Newcastle-upon-Tyne, UK \\ ${ }^{4}$ Maternal and Child Health, Burnet \\ Institute, Melbourne, Vic., Australia

\section{${ }^{*}$ Correspondence} \\ Theresa Lawrie, Evidence-Based Medicine \\ Consultancy Ltd, Bath, UK. \\ Email: tesslawrie@gmail.com

\section{Funding Information} \\ UNDP/UNFPA/UNICEF/WHO/World \\ Bank Special Programme of Research, \\ Development and Research Training in \\ Human Reproduction (HRP)
}

\begin{abstract}
Background: Several uterotonic options exist for prevention of postpartum hemorrhage (PPH); hence, cost-effectiveness is an important decision-making criterion affecting uterotonic choice.

Objective: To conduct a systematic review of cost-effectiveness of uterotonics for PPH prevention to support a WHO guideline update.

Search strategy: We searched major databases from 1980 to June 2018 and the National Health Services Economic Evaluation (NHS EED) database from inception (1995) to March 2015 for eligible studies.

Selection criteria: We included comparative economic evaluations, cost-utility analyses, and resource-utilization studies.

Data collection and analysis: Two reviewers independently assessed studies and extracted data organized by birth mode and setting.

Main results: We included 15 studies across all income categories that compared misoprostol versus no uterotonic (five studies) or versus oxytocin (one study), carbetocin versus oxytocin (eight studies), and one study comparing numerous uterotonics. In specific low-resource contexts, we found reasonably good evidence that misoprostol was cost-effective compared with no uterotonic. In the context of cesarean delivery, carbetocin was more cost favorable than oxytocin but certainty of this evidence was low. Conclusions: Evidence on the cost-effectiveness of various uterotonic agents was not generalizable. As the number of competing uterotonics increases, rigorous economic evaluations including contextual factors are needed.
\end{abstract}

\section{KEYWORDS}

Carbetocin; Cost-effectiveness; Ergometrine; Misoprostol; Oxytocin; Postpartum hemorrhage; Prostaglandins; Uterotonics

\section{1 | INTRODUCTION}

Postpartum hemorrhage (PPH) is generally defined as blood loss of $500 \mathrm{~mL}$ or more within 24 hours after birth and affects around $5 \%$ of women giving birth globally. ${ }^{1,2}$ It is most often caused by failure of the uterus to contract after childbirth. ${ }^{3}$ Pre-existing WHO guidance on $\mathrm{PPH}$ prevention recommends that all women giving birth are routinely offered an effective uterotonic agent during the third stage of labor. ${ }^{4}$

This is an open access article distributed under the terms of the Creative Commons Attribution IGO License which permits unrestricted use, distribution and reproduction in any medium, provided that the original work is properly cited. In any reproduction of this article there should not be any suggestion that WHO or the article endorse any specific organization or products. The use of the WHO logo is not permitted. This notice should be preserved along with the article's URL. (c) 2019 World Health Organization, licensed by International Federation of Gynecology and Obstetrics. 

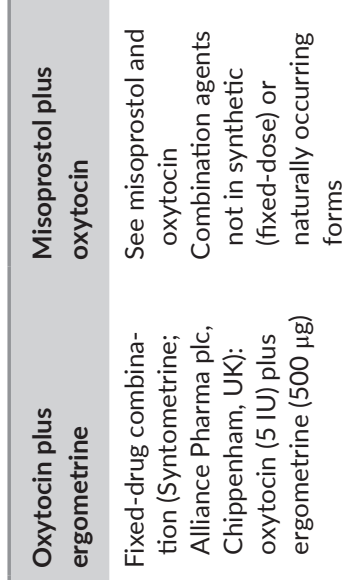

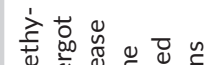

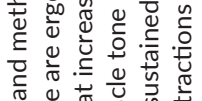

竞

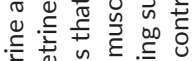

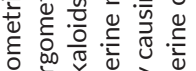

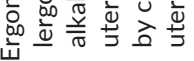

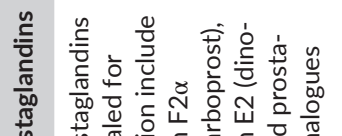

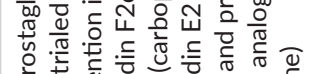

咅

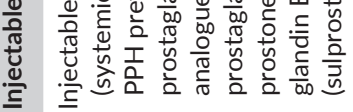

离 击

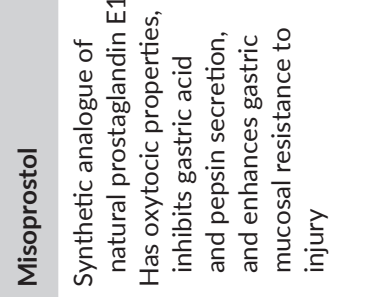

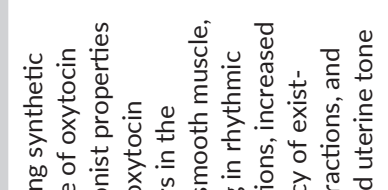

든

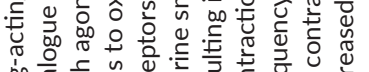

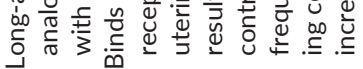

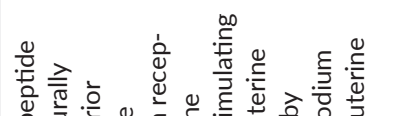

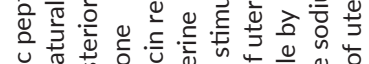

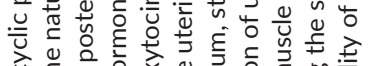

든

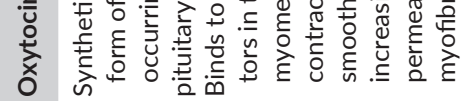

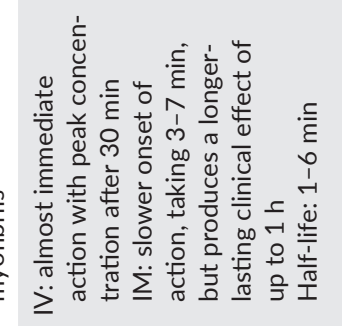

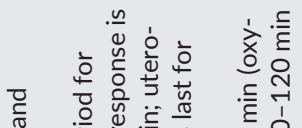

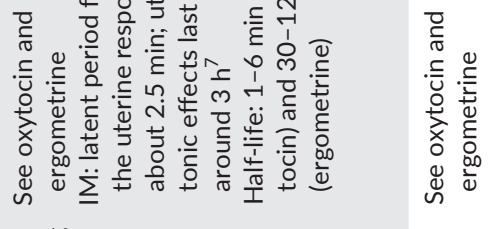

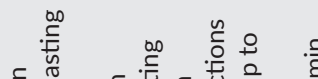

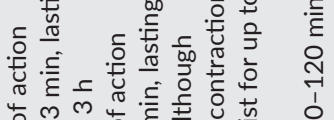

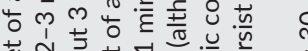

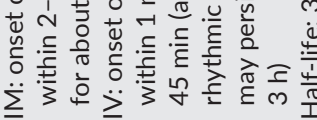

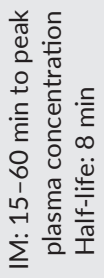

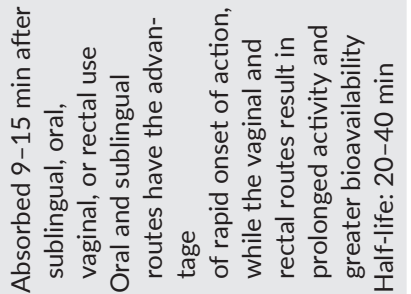

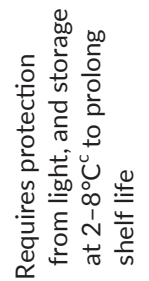
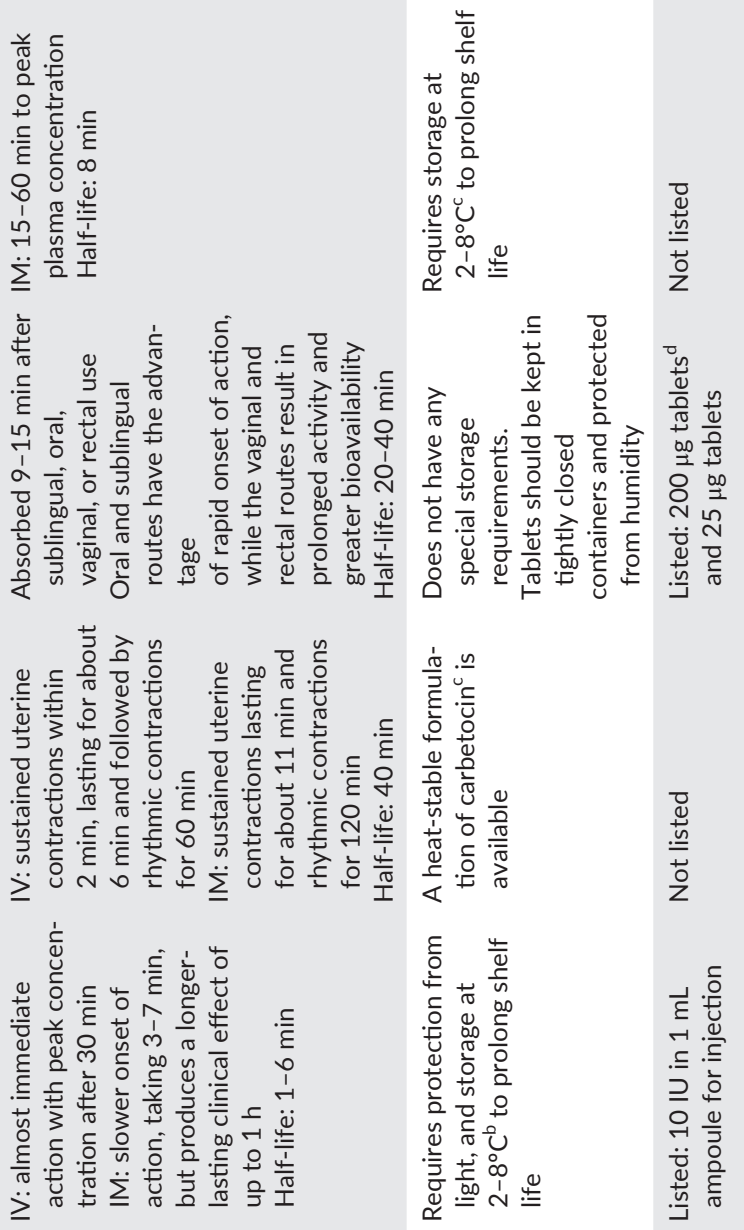

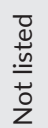

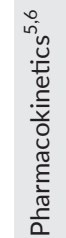

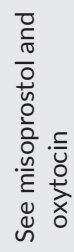

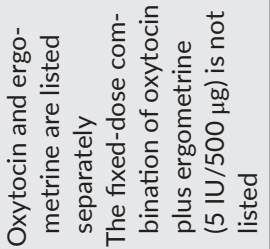

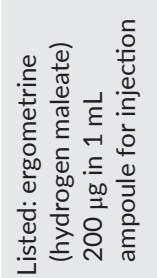

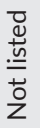

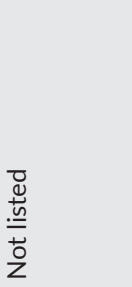

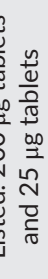
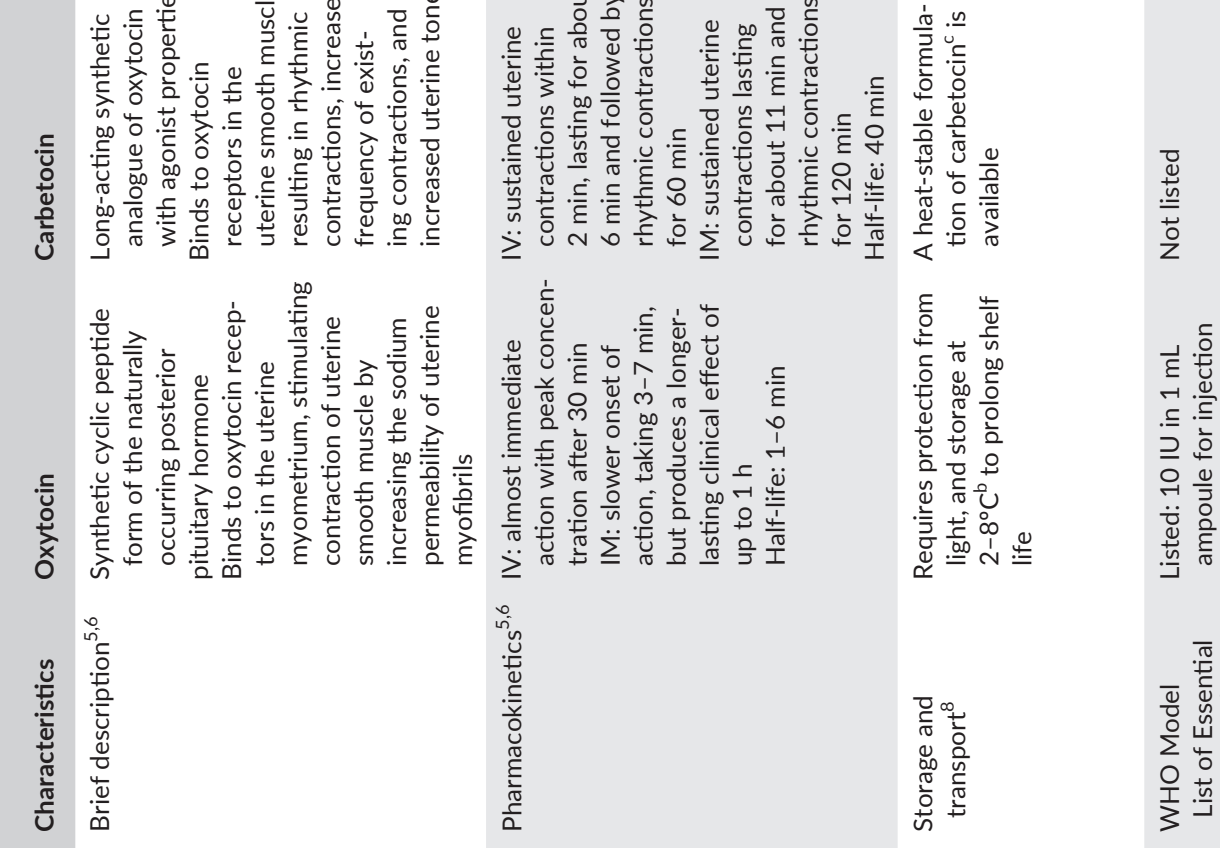

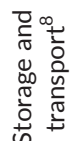

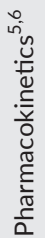

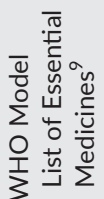

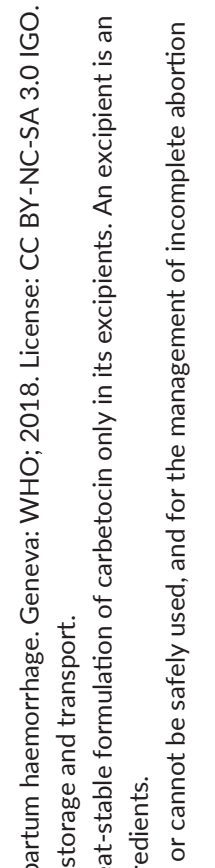

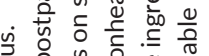

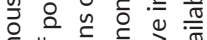

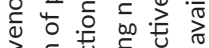

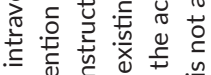

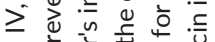

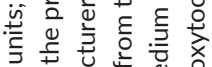

তั

年

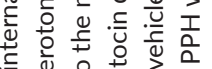

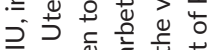

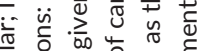

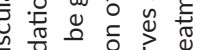

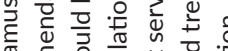

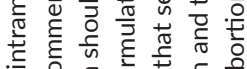

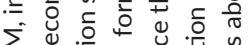

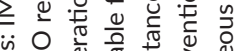

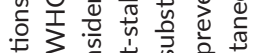

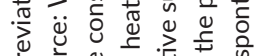

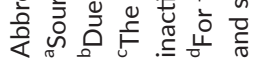


There are several uterotonic agents that are in clinical use or that have been evaluated for PPH prevention, including oxytocin, carbetocin, ergometrine, misoprostol, injectable prostaglandins (such as sulprostone and carboprost), as well as the combination agent Syntometrine (ergometrine plus oxytocin) (Alliance Pharma plc, Chippenham, UK) (Table 1). However, many settings (particularly lowresource settings) often do not have all uterotonic agents available. Since 2012, WHO has recommended oxytocin as the uterotonic of choice to prevent $\mathrm{PPH} .{ }^{4}$ In settings where oxytocin is not available, other uterotonics (ergometrine, ergometrine and oxytocin, or misoprostol) are the alternatives recommended by WHO.

When health systems' stakeholders consider which uterotonic option should be used in their context, consideration is given to a number of factors, including efficacy and safety, how feasible and acceptable the option is, whether it is cost-effective, and the resources required to provide it. The cost-effectiveness of a uterotonic option may differ depending on the mode of birth (vaginal birth or cesarean delivery) or birth setting (hospitals vs community settings) and, in theory, a high unit cost of a uterotonic option might potentially be offset by cost savings associated with a reduction in adverse outcomes.

While some studies have assessed cost-effectiveness of a single uterotonic in a specific setting, to our knowledge, no systematic review has been conducted to identify and assess the available economic evaluation evidence for all uterotonic options when used for PPH prevention. This review was performed in the context of an evidence base preparation to update WHO's recommendations on uterotonics for PPH prevention. ${ }^{10}$ The review aimed to summarize the available evidence on the cost-effectiveness of uterotonic agents when applied for the prevention of PPH. Additionally, we evaluated available evidence regarding which uterotonic agents are cost-effective to prevent PPH according to mode of birth (vaginal birth or cesarean delivery), and birth settings (community settings without skilled birth attendants, and in hospital settings where injectable uterotonic is not available).

\section{2 | MATERIALS AND METHODS}

\section{1 | Literature search}

We searched Medline (1980 to May week 4 2018), Embase (1980 to week 22 2018), and the National Health Services Economic Evaluation (NHS EED) database from inception in 1995 to 2 April 2015 (database closure) for studies evaluating costs and cost-effectiveness of any uterotonic agent, alone or in combination, in comparison with oxytocin, placebo, or another uterotonic agent used for the primary prevention of $\mathrm{PPH}$ in women in the third stage of labour in any setting (community, healthcare centre, or hospital).

\subsection{Data extraction and quality assessment}

Cochrane methodology for economic evaluations was used. ${ }^{11}$ Two researchers ( $T L$ and ER) independently assessed for inclusion all potentially eligible studies. Any disagreements were resolved through discussion or by consulting a third author. A data extraction form was adapted from the guidance for NHS EED abstracts of economic evaluations. ${ }^{12}$ For each included study, two authors independently extracted information on type of cost evaluation (study design), sources of effectiveness data, type of costs and their source, sources of outcome valuations, and type of adopted analytical approach. Where available, Incremental Cost-Effectiveness Ratios (ICERs) were extracted (an ICER is the ratio of the difference in costs between an intervention or treatment and a specified comparator to the difference in effectiveness or outcome between that intervention and the specified comparator). Quality of identified studies was systematically evaluated using the Consensus Health Economic Criteria (CHEC) checklist. ${ }^{13}$ Assessments were compared and disagreements resolved through discussion or consulting a third author.

\section{3 | Data synthesis}

We developed a conceptual framework on the possible cost consequences associated with use of a uterotonic agent to prevent PPH (Fig. 1). For identified studies, we tabulated the characteristics and findings of individual economic evaluations and supplemented it with a brief narrative summary of the findings. The currency and price year applicable to measures of costs in each original study were reported alongside measures of costs, incremental costs, and incremental cost-effectiveness. Findings were summarized according to mode of birth (vaginal birth or cesarean delivery) and birth setting (hospital or community).

\section{3 | RESULTS}

\subsection{Characteristics of included studies}

The combined deduplicated search yield was 180 records. We additionally identified two records (one conference abstract and one commissioned report) through searching the reference lists of included papers. Out of 182 retrieved citations, 168 studies were assessed as irrelevant on title and abstract screening and nine full text retrievals contained no cost-effectiveness data, leaving 15 studies that met the eligibility criteria of this review (Fig. 2). One unpublished report identified in the reference list of an included study could not be retrieved: two were conference abstracts and contained limited data. ${ }^{14,15}$

Included studies were conducted in Columbia, ${ }^{15}$ Ecuador, ${ }^{16}$ India, ${ }^{17,18}$ Malaysia, ${ }^{19}$ Mexico. ${ }^{14}$ Peru, $^{20}$ Senegal, $^{21}$ Tanzania, ${ }^{22}$ Uganda, ${ }^{23}$ and the UK. ${ }^{24-27}$ One of the studies was an international study with a hypothetical cohort. ${ }^{28}$ Eleven of these studies were conducted from 2011 to 2018 and four studies were conducted from 2007 to $2010 .{ }^{14,17,18,22}$ Out of the 15 studies, three studies were funded by pharmaceutical companies, ${ }^{16,20,27}$ six studies were funded by organizations and institutions, such as WHO, the Bill and Melinda Gates Foundation, Department for International Development, National Institute for Health Research, and others, ${ }^{17,18,21,22,24,28}$ three studies were not funded, ${ }^{23,25,26}$ in two studies (abstracts) it was unclear whether they were funded or not, ${ }^{14,15}$ and one study did not disclose funding sources. ${ }^{19}$ 


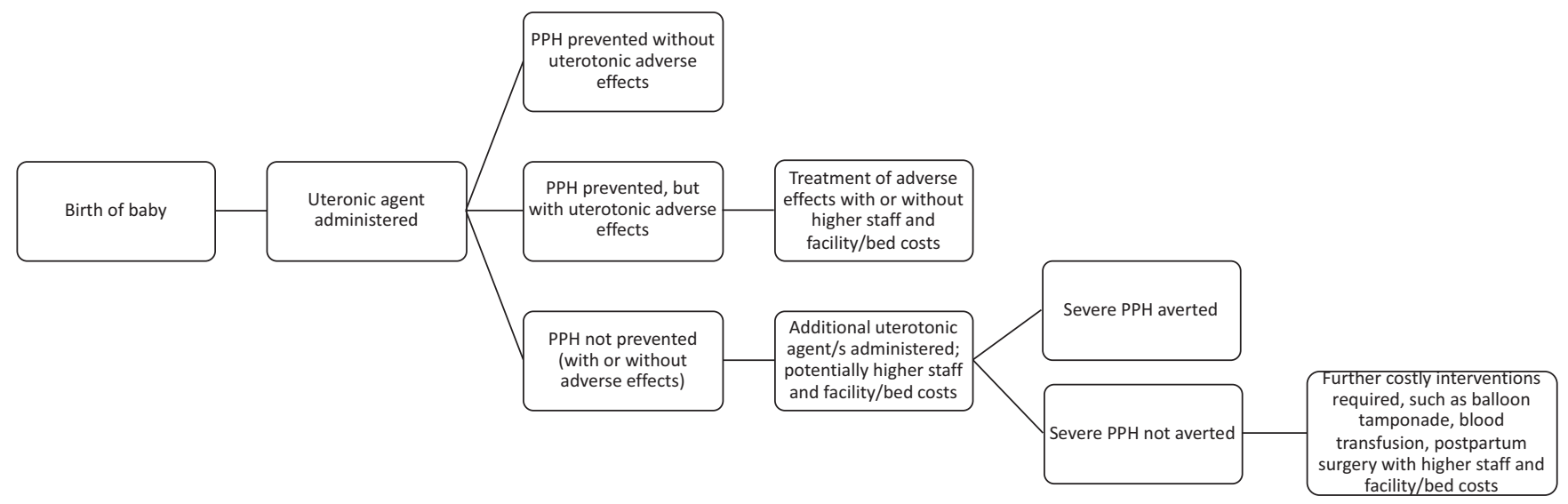

Increasing costs

FIGURE 1 Diagram of possible consequences associated with use of a uterotonic agent.

The relevant healthcare perspective was used in all studies except in one study, which adopted a WHO perspective. A majority of the studies were model-based, using decision analytical models (decision trees), with the exception of two studies that used data directly obtained from randomized controlled trials, ${ }^{14,21}$ one observational study, ${ }^{25}$ and a service composite clinical and financial analysis study. ${ }^{27}$ Various measures (condition-specific and generic) were used to measure health outcome of uterotonics including incidence and cases of $\mathrm{PPH}$, use of additional uterotonics, mortality, probability of mortality, referral to higher-level health facility, adverse effects, quality-adjusted life years (QALYs), and disability-adjusted life years (DALYs). One study was a cost-effectiveness analysis of several uterotonics based on a network meta-analysis (NMA) adopting a UK perspective. ${ }^{24}$ Six studies evaluated the cost-effectiveness of misoprostol versus third stage management without any uterotonics (five studies) or oxytocin (one study), all of which were conducted in settings with low access to facility births. ${ }^{17,18,21-23,28}$ A further eight studies evaluated the cost-effectiveness of carbetocin versus oxytocin across various facility settings. ${ }^{14-16,19,20,24-26}$

\subsection{Which uterotonic agents are cost-effective for preventing PPH at vaginal birth?}

One study based on the UK health service perspective provided evidence on this question. The study was a cost-effectiveness analysis conducted as part of a systematic review and NMA that compared the different uterotonics and ranked them according to cost and effectiveness (conducted as part of a UK health technology assessment). ${ }^{24}$ When adverse events were considered, oxytocin (which ranked fourth in effectiveness) was the least costly uterotonic agent. Compared with oxytocin, carbetocin (which ranked as a more effective agent) was associated with an ICER of approximately US \$1193.58 per additional PPH of greater than or equal to $500 \mathrm{~mL}$ avoided, and US \$29 464.19 per additional PPH greater than or equal to $1000 \mathrm{~mL}$ avoided. These findings were similar for hospital and community settings in the UK.
Misoprostol was found to be the costliest uterotonic agent in this setting when adverse events were considered.

\subsection{Which uterotonic agents are cost-effective for preventing PPH at cesarean delivery?}

Eight cost-effectiveness analyses and one service evaluation study contributed data. ${ }^{14-16,19,20,24-27}$ Four were from a high-income country (UK) and five were from middle-income countries (Columbia, Ecuador, Malaysia, Mexico, and Peru). Apart from the UK-based study that evaluated several uterotonic options, ${ }^{24}$ all studies compared carbetocin $(100 \mu \mathrm{g})$ to oxytocin (5 or $10 \mathrm{IU}$, if dose was reported). Four studies based their cost-effectiveness analysis on effectiveness outcome data of a published Cochrane systematic review of carbetocin for preventing $\mathrm{PPH},{ }^{16,19,20,26}$ three evaluations were based on prospective studies, ${ }^{14,25,27}$ one on the findings of the NMA, ${ }^{24}$ and the source of the effectiveness data in one study reported as a conference abstract was unclear. ${ }^{15}$

In the UK cost-effectiveness analysis that compared various uterotonic options, ${ }^{24}$ data on costs for adverse events (considered in this model to be nausea, vomiting, hypertension, headache, tachycardia, shivering, and fever) were described by investigators as very uncertain. However, when these were considered, carbetocin was associated with lower costs relative to the next most cost-effective option-the misoprostol plus oxytocin combination. The ICER for averting one case of PPH greater than or equal to $1000 \mathrm{~mL}$ in the UK context was reported as approximately US $\$ 2927.30$ with carbetocin versus the misoprostol plus oxytocin combination, and averting a major adverse outcome was US \$114 347.78. However, when adverse events were not considered, the misoprostol plus oxytocin combination was found to be more cost-effective than carbetocin. In these analyses, these two uterotonic options (i.e. carbetocin or misoprostol plus oxytocin) were both more cost-effective than misoprostol or oxytocin alone.

The eight other studies that evaluated economic outcomes associated with carbetocin at cesarean delivery all compared carbetocin 


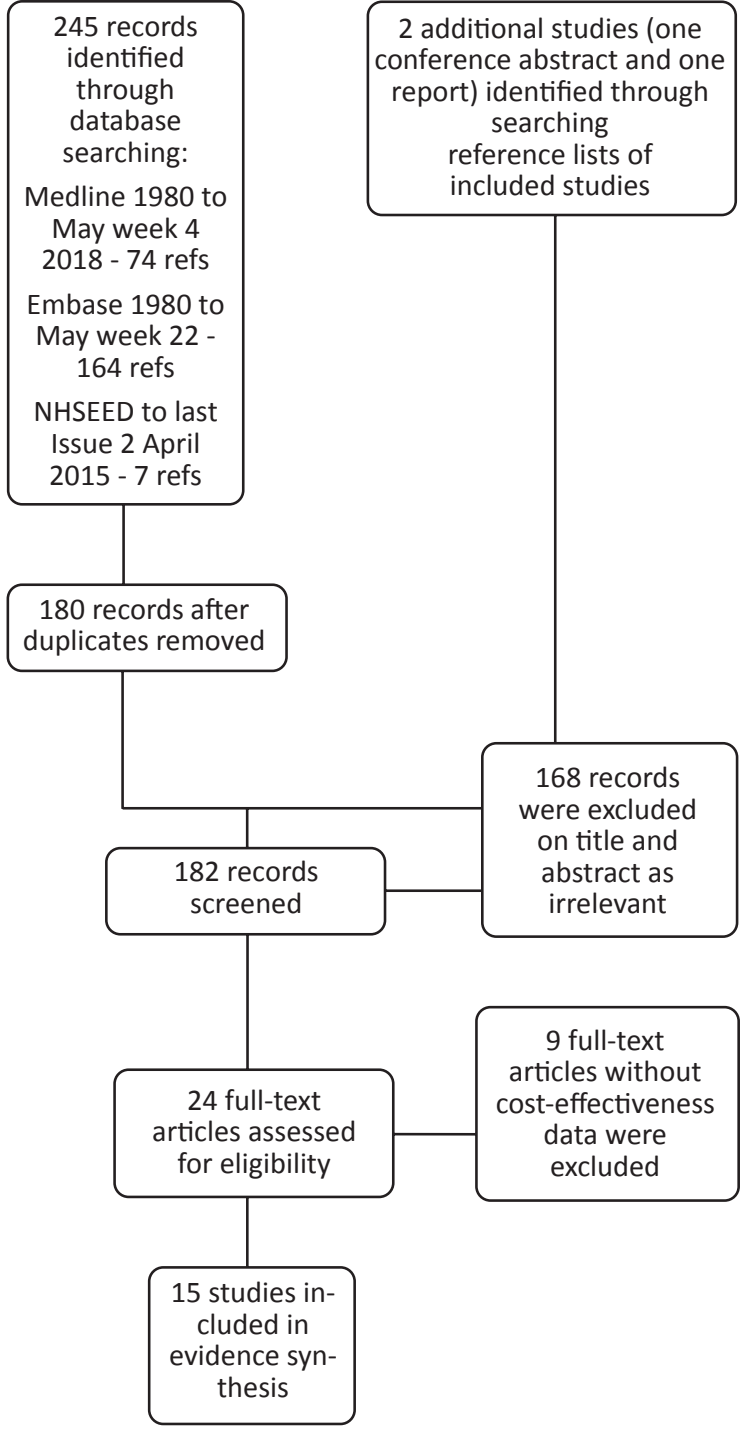

FIGURE 2 Flow diagram of search results and study selection.

$(100 \mu \mathrm{g})$ to oxytocin (5 or $10 \mathrm{IU}$, where dose was reported). Seven studies concluded that carbetocin was cost-effective at cesarean delivery compared with oxytocin. ${ }^{14-16,19,20,25,26}$ However, in some of these studies ${ }^{19,20,26}$ investigators reported that uncertainty around costs and other input data made it difficult to evaluate costeffectiveness with any certainty. We considered the quality of the evidence provided by these studies to be low, owing to a lack of sensitivity analyses and incomplete description of the methods of outcome and costs measurements in most studies. In addition, at least two of the studies from middle-income countries favoring carbetocin were funded by the manufacturer and, as such, we interpreted findings from these studies and those of the conference abstracts with limited data ${ }^{14,15}$ cautiously owing to the potential risk of bias and imprecision.

In the remaining study, a UK service evaluation study, ${ }^{27}$ investigators reported a significant increase in the cost of care during the period from birth of baby to transfer to the postnatal ward of low-risk women undergoing elective cesarean delivery (from approximately
US $\$ 104.27$ before the introduction of carbetocin, to US $\$ 128.35$ following this $(P<0.01)$, but economic modelling was not performed in this study.

\subsection{Uterotonic agents in community settings without skilled birth attendants}

The cost-effectiveness of misoprostol was evaluated in settings with low access to modern birth facilities (lack of skilled birth attendants, inadequate transport and storage facilities, or oxytocin not available) in six studies. ${ }^{17,18,21-23,28}$ These studies were of moderate to high quality according to the CHEC checklist, ${ }^{13}$ and most used a model-based approach to estimate incremental costs of introducing misoprostol to prevent PPH in these settings. Four studies evaluated misoprostol as a 600- $\mu$ g dose (oral or sublingual), ${ }^{17,18,21,23}$ one used a $200-\mu \mathrm{g}$ dose, ${ }^{28}$ and one used a 1000$\mu \mathrm{g}$ dose rectally administered. ${ }^{22}$ In most studies administration of misoprostol was undertaken by lay health workers; in one study in Uganda it was distributed prenatally to pregnant women for selfadministration following birth. ${ }^{23}$ Although cost-effectiveness measures and reporting differed (e.g. ICER per case of PPH avoided, per DALY gained, per life saved, cost savings per 1000 births, etc.), findings across studies consistently showed that misoprostol was highly cost-effective (or led to cost savings) compared to no uterotonic agents in these settings. A study from Senegal, ${ }^{21}$ found misoprostol (600 $\mu \mathrm{g}$ orally) to be more cost-effective than oxytocin (10 IU provided via the Uniject system; BD Company, Franklin Lakes, NJ, USA) in rural settings; it should be noted that the finding may be driven mainly by the lower cost of misoprostol. Oxytocin was assumed to have higher wastage costs based on a rate derived from a randomized trial in which $12.1 \%$ of oxytocin Uniject devices were discarded owing to breakage, being compromised by heat, or having passed the expiration date. ${ }^{29}$

\subsection{Uterotonic agents in hospital settings where oxytocin quality cannot be guaranteed}

We found no research evidence that directly addresses this question. However, in the UK NMA cost-effectiveness analysis, if the option of oxytocin alone or in combination with other agents was removed from the study analyses, findings suggest that carbetocin might be the most cost-effective uterotonic for vaginal birth (and cesarean delivery) in the UK context, followed by ergometrine (if adverse events were considered), or misoprostol (if adverse events were not considered). These findings were uncertain and might not be generalizable to other settings, particularly settings where injectable uterotonics cannot be used.

\section{4 | DISCUSSION}

Cost-effectiveness studies on PPH prevention in different contexts were sparse given the range of uterotonic agents available. We found 


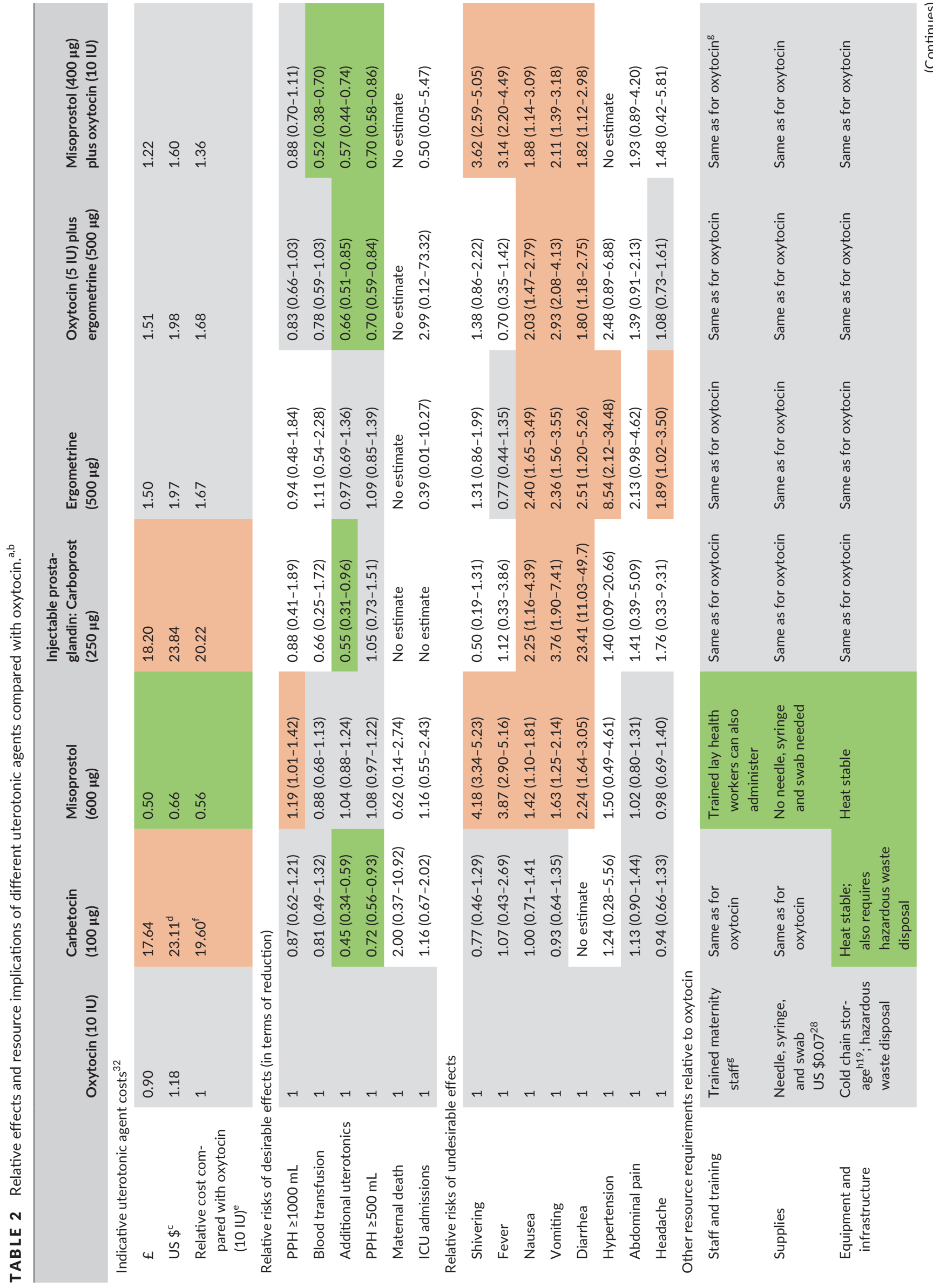




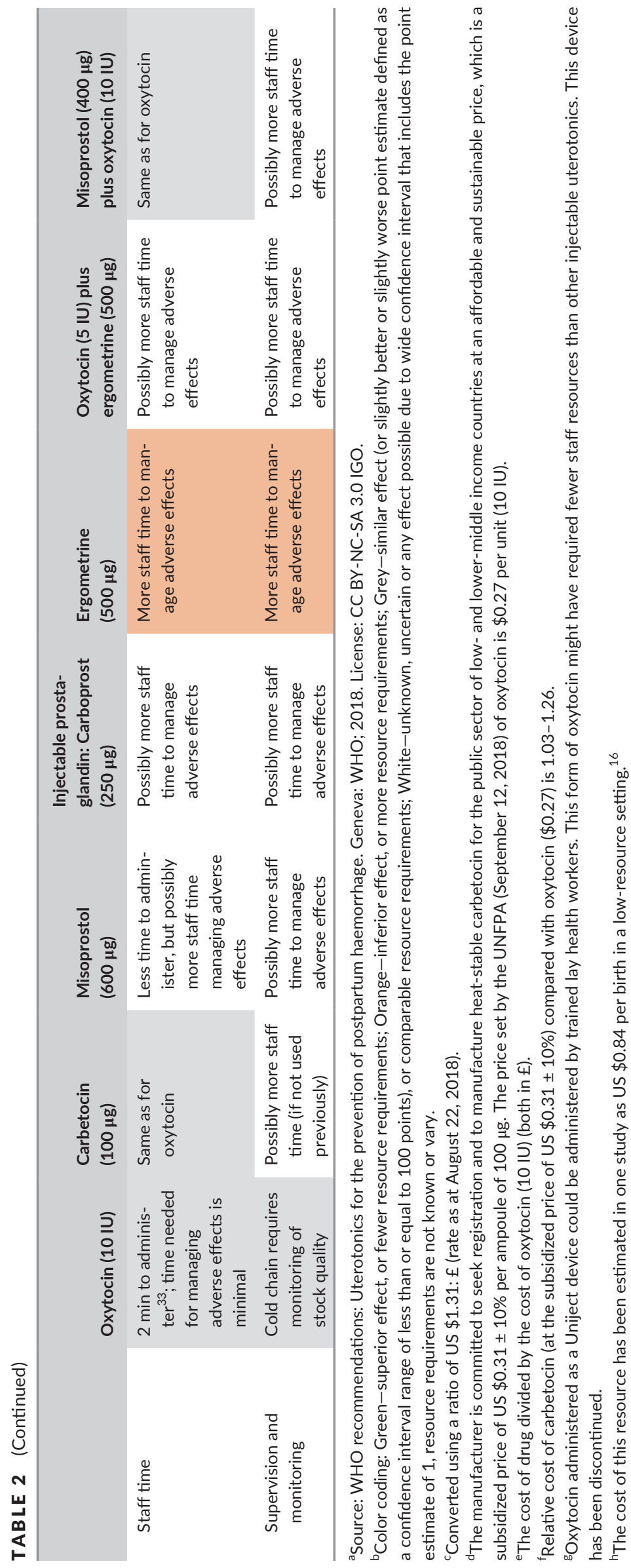


reasonable quality evidence that misoprostol compared with no uterotonic agent was cost-effective for preventing PPH at vaginal birth in settings with low access to modern birth facilities. With regard to preventing $\mathrm{PPH}$ at cesarean delivery, the evidence suggested that carbetocin might be more cost-effective than oxytocin, and that the next most cost-effective option after carbetocin might be the combination of misoprostol and oxytocin; however, these findings were fairly uncertain.

To our knowledge this is the only systematic review of the costeffectiveness of uterotonic agents for PPH prevention to date. We used a broad, systematic search to identify potentially eligible studies, and we were guided by principles and methods of the Cochrane Handbook and the NHS EED. Unfortunately, review findings were often uncertain owing to methodological limitations and the quality of reporting in the included studies. For vaginal birth, we found only two studies comparing cost-effectiveness of one uterotonic option with another uterotonic option, and no studies comparing cost-effectiveness of multiple uterotonics in low- and middle-income (LMIC) country settings.

Another limitation is that the evidence derived from a rigorous cost-effectiveness analysis conducted in the UK that compared various uterotonic options with each other was probably not generalizable to many other countries. While this study obtained a high score on the CHEC checklist, ${ }^{13}$ input cost data (such as bed, staff, and intervention costs) are likely to be considerably different in LMICs. In addition, in this study, misoprostol was associated with higher costs, which were derived from longer bed stays and unnecessary tests related to its common adverse effects (shivering and fever); such costs might be negligible in LMIC settings, owing to expectant management of these adverse effects and lower bed costs.

Heat-stable uterotonics that do not require refrigeration (such as misoprostol and the heat-stable formulation of carbetocin) can offer advantages for low-resource settings with no or limited access to cold chain transport and storage. While the evidence suggests that misoprostol is cost-effective in these settings, little is known about the cost-effectiveness of carbetocin in this context or its relative costeffectiveness compared with other options for vaginal birth in different settings. It should be noted that carbetocin requires a trained, skilled health provider to administer it parenterally, whereas misoprostol can be administered orally by lower cadres of health workers (such as community health workers or lay health workers). In addition, as the price of the uterotonic agent appears to be critical to costing models for lowresource settings (more so than for high-resource setting models), the cost-effectiveness of carbetocin relative to misoprostol will be strongly affected by the supply prices. While the price range across studies included in the review was US $\$ 13.10-25.60$ per $100-\mu$ g carbetocin

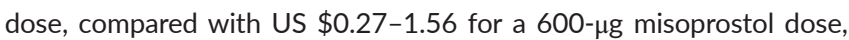
we note that the manufacturer of heat-stable carbetocin has made an in-principle commitment to make the heat-stable formulation of carbetocin available in the public sector of LMICs at an affordable and sustainable price, comparable to the UNFPA price of oxytocin (US \$0.27). ${ }^{30}$

This study is a systematic review of available formal economic analyses of uterotonics agents but is itself not an economic evaluation or analysis. Findings from our review highlight the lack of reliable evidence on the cost-effectiveness of these agents in different settings, and hence a well-conducted economic evaluation of all agents (particularly in low-resource settings) is needed. Such an analysis would serve as an important guide to decision and policy making in PPH prevention, particularly in LMICs.

Given the paucity of available evidence on uterotonic costeffectiveness, for the purposes of informing the WHO guideline update process, we created a logic model on cost-effectiveness considerations across the different uterotonic options to aid decision makers and other stakeholders (Table 2). In this approach, we used up-to-date evidence on the relative effectiveness and safety of the different uterotonic agents relative to oxytocin from an updated Cochrane systematic review and NMA. ${ }^{31}$ We tabulated the risk ratios of desirable and undesirable effects. In addition, we tabulated their potential cost consequences relative to oxytocin, including resource requirements related to staffing and training, equipment and infrastructure, staff time, supplies, and supervision and monitoring that are associated with these options (Table 2). For uterotonic supply prices, we referenced costs from the British National Formulary, ${ }^{32}$ which served to illustrate relative drug costs in a setting where all agents were available. The intention of the logic model was to facilitate a systematic, qualitative assessment of resource use and cost-effectiveness factors across the different uterotonic options in the absence of good evidence of the relative cost-effectiveness of available uterotonics in different contexts.

In conclusion, evidence on the cost-effectiveness of various uterotonic agents is sparse and largely not generalizable to different contexts. In the absence of reliable evidence, it is likely that the choice of uterotonic will be highly influenced by uterotonic price, as well as contextual factors. In view of the increasing number of competing uterotonics, more rigorous economic evaluations based on robust efficacy evidence and considerations of contextual factors are needed.

\section{AUTHOR CONTRIBUTIONS}

TL drafted the protocol with input from OO, JV, and ER. TL, ER, and PS screened studies and extracted data. TL led the writing of the manuscript, with input from JV, ER, PS, OO, and LT. All authors reviewed and approved the final version.

\section{ACKNOWLEDGMENTS}

This review was funded by the UNDP/UNFPA/UNICEF/WHO/World Bank Special Programme of Research, Development and Research Training in Human Reproduction (HRP), a cosponsored program executed by the WHO. The manuscript represents the views of the named authors only.

\section{CONFLICTS OF INTEREST}

The authors have no conflicts of interest to declare. 


\section{REFERENCES}

1. Souza JP, Gülmezoglu AM, Vogel J, et al. Moving beyond essential interventions for reduction of maternal mortality (the WHO Multicountry Survey on Maternal and Newborn Health): A crosssectional study. Lancet. 2013;381:1747-1755.

2. Carroli G, Cuesta C, Abalos E, et al. Epidemiology of postpartum haemorrhage: A systematic review. Best Pract Res Clin Obstet Gynecol. 2008;22:999-1012.

3. Say L, Chou D, Gemmill A, et al. Global causes of maternal death: A WHO systematic analysis. Lancet Global Health. 2014;2:e324-e333.

4. World Health Organization. WHO Recommendations for the Prevention and Treatment of Postpartum Haemorrhage. Geneva: WHO; 2012. https ://www.who.int/reproductivehealth/publications/maternal_perin atal_health/9789241548502/en/. Accessed November 27, 2018.

5. Wishart DS, Feunang YD, Guo AC, et al. DrugBank 5.0: A major update to the DrugBank database for 2018. Nucleic Acids Res. 2018;46(D1):D 1074-D1082.

6. Kim S, Thiessen PA, Bolton EE, et al. PubChem Substance and Compound databases. Nucleic Acids Res. 2016;44(D1):D1202-D1213.

7. Electronic Medicines Compendium (eMC). Syntometrine ampoules. [Website] https://www.medicines.org.uk/emc/product/865/smpc. 2016. Accessed November 27, 2018.

8. World Health Organization. The International Pharmacopoeia. Geneva [WHO Website]. 2017. http://apps.who.int/phint/en/p/ docf/. Accessed November 27, 2018.

9. World Health Organization. Report of the WHO Expert Committee on Selection and Use of Essential Medicines. Geneva [WHO Website]. 2017. http://www.who.int/selection_medicines/committees/exper t/20/en/. Accessed November 27, 2018.

10. World Health Organization. Executive Guideline Steering Group for Updating WHO Maternal and Perinatal Health Recommendations (2017-2019) [WHO Website]. 2017. http://www.who.int/repro ductivehealth/publications/updating-mnh-recommendations/en/. Accessed November 27, 2018.

11. Higgins JPT, Green S, eds. Economics and economic evaluation. In: The Cochrane Handbook for Systematic Reviews of Interventions. Oxford: The Cochrane Collaboration; 2011.

12. Craig D, Rice S. CRD Report 6: NHS Economic Evaluation Database Handbook, 3rd edn. York, UK: Centre for Reviews and Dissemination, University of York; 2007.

13. Evers $\mathrm{S}$, Goossens $\mathrm{M}$, de Vet $\mathrm{H}$, et al. Criteria list for assessment of methodological quality of economic evaluations: Consensus on Health Economic Criteria. Int J Technol Assess Health Care. 2005;21:240-245.

14. Del Angel-Garcia G, Garcia-Contreras F, Constantino-Casas P, et al. Economic evaluation of carbetocin for the prevention of uterine atony in patients with risk factors in Mexico. Value Health. 2006;9:A254.

15. Rueda C, Caceres LA, Caicedo A. Cost effectiveness of carbetocin compared with oxytocin to prevent postpartum hemorrhage due to uterine atony in patients with risk factors [In Spanish]. Value Health 2013;16:A709-A710.

16. Henriquez-Trujillo AR, Lucio-Romero RA, Bermudez-Gallegos $K$ Analysis of the cost-effectiveness of carbetocin for the prevention of hemorrhage following cesarean delivery in Ecuador. Future Virol. 2017;12:529-536.

17. Sutherland T, Meyer C, Bishai DM, et al. Community-based distribution of misoprostol for treatment or prevention of postpartum hemorrhage: Cost-effectiveness, mortality, and morbidity reduction analysis. Int J Gynecol Obstet. 2010;108:289-294.

18. Sutherland T, Bishai DM. Cost-effectiveness of misoprostol and prenatal iron supplementation as maternal mortality interventions in home births in rural India. Int J Gynecol Obstet. 2009;104:189-193.

19. Voon HY, Shafie AA, Bujang MA, et al. Cost effectiveness analysis of carbetocin during cesarean section in a high volume maternity unit. $J$ Obstet Gynecol Res. 2018;44:109-116.

20. Caceda SI, Ramos RR, Saborido CM. Pharmacoeconomic study comparing carbetocin with oxytocin for the prevention of hemorrhage following cesarean delivery in Lima, Peru. J Comparative Effectiveness Res. 2018;7:49-55.

21. Vlassoff M, Diallo A, Philbin J, et al. Cost-effectiveness of two interventions for the prevention of postpartum hemorrhage in Senegal. Int J Gynecol Obstet. 2016;133:307-311.

22. Bradley SE, Prata N, Young-Lin N, Bishai DM. Cost-effectiveness of misoprostol to control postpartum hemorrhage in low-resource settings. Int J Gynecol Obstet. 2007;97:52-56.

23. Lubinga SJ, Atukunda EC, Wasswa-Ssalongo G, et al. Potential costeffectiveness of prenatal distribution of misoprostol for prevention of postpartum hemorrhage in Uganda. PLoS ONE. 2015;10:e0142550.

24. Gallos ID, Williams H, Price MJ, et al. Uterotonic drugs to prevent postpartum hemorrhage: A network meta-analysis. Health Technol Assess. 2019;23:1-356.

25. Luni Y, Borakati A, Matah A, et al. A prospective cohort study evaluating the cost-effectiveness of carbetocin for prevention of postpartum haemorrhage in caesarean sections. J Obstet Gynecol. 2017;37:601-604

26. van der Nelson HA, Draycott T, Siassakos D, et al. Carbetocin versus oxytocin for prevention of post-partum haemorrhage at caesarean section in the United Kingdom: An economic impact analysis. Eur J Obstet Gynecol Reprod Biol. 2017;210:286-291.

27. Higgins L, Mechery J, Tomlinson AJ. Does carbetocin for prevention of postpartum haemorrhage at caesarean section provide clinical or financial benefit compared with oxytocin? J Obstet Gynecol. 2011:31:732-739.

28. Lang DL, Zhao FL, Robertson J. Prevention of postpartum haemorrhage: Cost consequences analysis of misoprostol in low-resource settings. BMC Pregnancy Childbirth. 2015;15:305.

29. Diop A, Daff B, Sow M, et al. Oxytocin via Uniject (a prefilled singleuse injection) versus oral misoprostol for prevention of postpartum haemorrhage at the community level: A cluster-radomised controlled trial. Lancet Global Health. 2016;4:e37-e344.

30. Widmer M, Piaggio G, Nguyen TMH, et al. Heat-stable carbetocin versus oxytocin to prevent hemorrhage after vaginal birth. $N$ Engl $J$ Med. 2018;379:743-752.

31. Gallos ID, Williams HM, Price MJ, et al. Uterotonic agents for preventing postpartum haemorrhage: A network meta-analysis. Cochrane Database Syst Rev. 2018;(4):CD011689.

32. Joint Formulary Committee. British National Formulary 72 (September 2016-March 2017). London: BMJ Group; 2016.

33. OneHealth Model: intervention treatment assumptions (draft 28 September). Geneva and Glastonbury, CT: United Nations InterAgency Working Group on Costing and the Futures Institute; 2013. https:// avenirhealth.org/Download/Spectrum/Manuals/Intervention\%20 Assumptions\%202013\%209\%2028.pdf. Accessed November 27, 2018. 